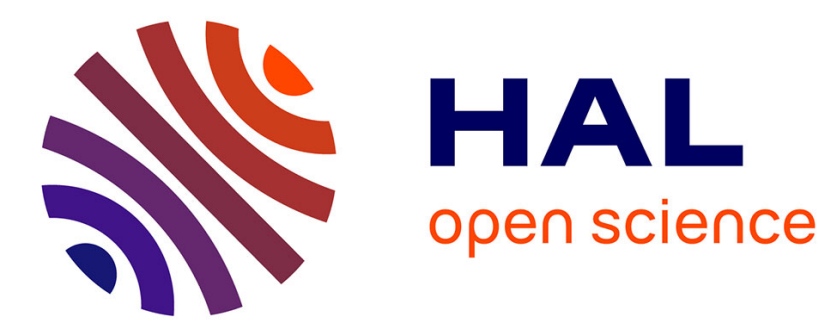

\title{
Temporal and Polarization Symmetry Breaking in Ring Resonators
}

\author{
F. Copie, M. Woodley, L. del Bino, J. M Silver, S. Zhang, P. Del'haye
}

\section{To cite this version:}

F. Copie, M. Woodley, L. del Bino, J. M Silver, S. Zhang, et al.. Temporal and Polarization Symmetry Breaking in Ring Resonators. Frontiers in Optics, 2018, Washington, France. pp.JW4A.32, 10.1364/FIO.2018.JW4A.32 . hal-02406861

\section{HAL Id: hal-02406861 \\ https://hal.science/hal-02406861}

Submitted on 12 Dec 2019

HAL is a multi-disciplinary open access archive for the deposit and dissemination of scientific research documents, whether they are published or not. The documents may come from teaching and research institutions in France or abroad, or from public or private research centers.
L'archive ouverte pluridisciplinaire HAL, est destinée au dépôt et à la diffusion de documents scientifiques de niveau recherche, publiés ou non, émanant des établissements d'enseignement et de recherche français ou étrangers, des laboratoires publics ou privés. 


\title{
Temporal and Polarization Symmetry Breaking in Ring Resonators
}

\author{
F. Copie," M. T. M. Woodley, L. Del Bino, J. M. Silver, S. Zhang, and P. Del'Haye ${ }^{\dagger}$ \\ National Physical Laboratory (NPL), Teddington TW11 OLW, UK \\ francois.copie@npl.co.uk \\ tpascal.delhaye@npl.co.uk
}

\begin{abstract}
We study the occurrence of two symmetry breaking processes in ring resonators pumped by short pulses. Different scenarios are predicted depending on the input characteristics, including a complex dynamical interplay between the two mechanisms.
\end{abstract}

OCIS codes: (190.3270) Kerr effect; (230.5750) Resonators; (230.4320) Nonlinear optical devices.

Nonlinear optical resonators are currently the subject of intense studies aiming at a better understanding of their dynamics both for fundamental research and applied aspects in fields such as spectroscopy, ranging, all-optical data storage or metrology. Among other features, optical ring resonators have been found to exhibit several processes of symmetry breaking which tend to make their behaviour more complex. For instance, breaking of the symmetry between two counter-propagating beams has been observed [1-3] as well as between fields of different polarization [4] or in the temporal shape of the intracavity field [5]. In this study we identify, for the first time to our knowledge, a regime for which two of these distinct symmetry breaking mechanisms coexist in a ring cavity. We numerically investigate the interplay between polarization and temporal symmetry breaking which appears to greatly depend on experimentally controllable parameters.

We consider a 1D isotropic passive ring cavity made of a dispersive medium exhibiting a Kerr nonlinearity (see Fig. 1(a)) pumped in the anomalous dispersion regime. Light propagation in this system can be described by the following set of two coupled normalized Lugiato-Lefever equations [6]:

$$
\begin{aligned}
& \frac{\partial E_{+}}{\partial z}-i\left[\frac{1}{3}\left|E_{+}\right|^{2}+\frac{2}{3}\left|E_{-}\right|^{2}+\frac{1}{2} \frac{\partial^{2}}{\partial \tau^{2}}\right] E_{+}+(1+i \Delta) E_{+}=S_{+}(\tau) \\
& \frac{\partial E_{-}}{\partial z}-i\left[\frac{1}{3}\left|E_{-}\right|^{2}+\frac{2}{3}\left|E_{+}\right|^{2}+\frac{1}{2} \frac{\partial^{2}}{\partial \tau^{2}}\right] E_{-}+(1+i \Delta) E_{-}=S_{-}(\tau)
\end{aligned}
$$

where $E_{+}$and $E_{-}$describe the left and right circularly polarized components of the intracavity field respectively, $S_{+}$and $S_{-}$the corresponding input fields, and $\Delta$ is the cavity detuning (difference of frequency between the pump and a resonance of the cavity). From these equations one can see that each field undergoes a cross-phase modulation due to the presence of the field of opposite handedness which is twice as strong as the self-phase modulation. This is at the origin of the polarisation symmetry breaking (PSB) mechanism in the system. In our study, we consider that the cavity is synchronously pumped by short Gaussian-shaped pulses that can entail the breaking of the temporal symmetry (TSB) of the intracavity field with respect to the input field.
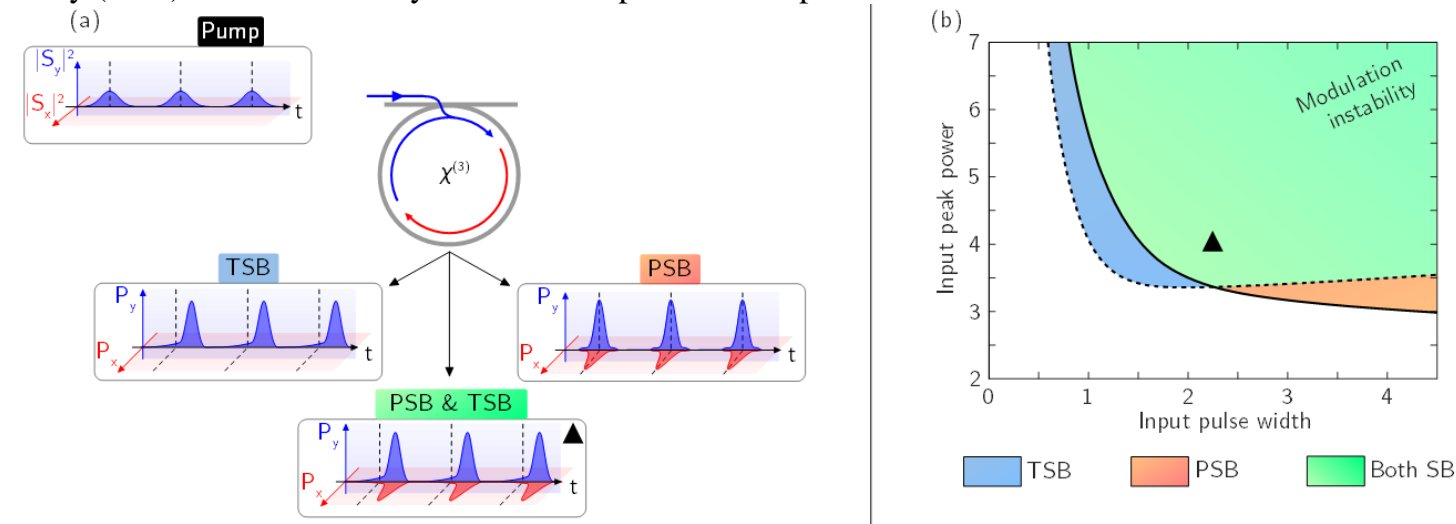

Fig. 1. (a) Schematic of the different types of symmetry breaking in a ring resonator synchronously pumped by short pulses. (b) Map of the different operating regimes as a function of the input parameters (peak power and pulse width) obtained numerically. The black triangle refers to the results presented in Fig. 2. PSB: polarization symmetry breaking, TSB: temporal symmetry breaking. 
We numerically studied Eqs. (1) and (2) by performing extensive simulations of the resonance scanning from negatively (red) to positively (blue) detuned over a wide range of input parameters (peak power and pulse width). This allowed us to build a map of the different operating regimes which is presented in Fig. 1(b). We identified ranges of parameters for which only PSB or TSB occurs (highlighted in red or blue respectively). More interestingly, over a large range of parameters both mechanisms can arise, resulting in a complex interplay (green region). Note that modulation instability is susceptible to occur for large input powers and leads to the breakup of the intracavity field into multiple pulses which does not allow to identify unambiguously the role of TSB. To obviate this effect, we thus focussed our attention on the behaviour of the system close to the symmetry breaking thresholds (see the black triangle).

Figure 2 illustrates results obtained for pump parameters in the region where both symmetry breaking occurs and for two different detuning scanning rates. For an arbitrarily slow scanning rate (left column) the power of the intracavity field polarized along the $y$-axis at the fast time corresponding to the maximum of the input power $(\tau=0)$ increases when approaching the resonance (Fig. 2(a)). At some point, PSB occurs, translating into the generation of an $x$-polarized component (see Fig. 2(c)) just before TSB arise (see Fig. 2(b)). This in turn reduces the PSB. Further on in the scanning, the temporal symmetry is retrieved which allows for a new increase of the PSB before it vanishes. Finally, the system jumps out of the resonance when $\Delta$ exceeds 4.5 . The same simulation realized with a scanning rate five times faster (right column) shows similar results except that no sign of TSB is observed. This emphasise that the interplay between the two processes depends on the dynamics of the input field (sweeping speed of the pump frequency) which can be tuned in experiment.
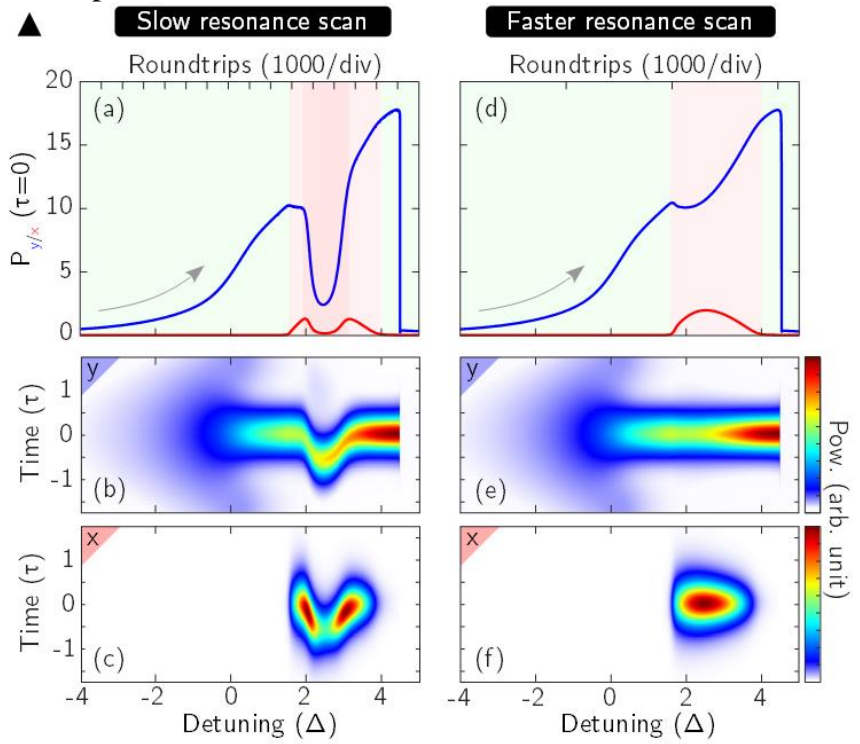

Fig. 2. Dynamics of the interplay between PSB and TSB. (a,d) Round-trip-to-round-trip evolution of the intracavity power of the $x$ - and $y$ polarized components (red and blue respectively) at the fast time corresponding to the maximum of the input power $(\tau=0)$ for two different detuning scanning rates. Regions where one or both of the symmetries are broken are highlighted by a red background. (b,c,e,f) $2 \mathrm{D}$ color plots of the evolution of the intracavity pulses separated in $x$ - and $y$ - polarized components for both detuning scanning rates. The input field is linearly polarized along the $y$-axis. Detuning scanning rate is $5.6 \times 10^{-4} \mathrm{rad} /$ round-trip for the left column and $2.8 \times 10^{-3} \mathrm{rad} / \mathrm{round}$-trip for the right one.

To conclude, we studied an original operating regime of isotropic nonlinear ring resonators pumped by short pulses. The isotropic nature of the cavity induces a cross-phase modulation between two counter-rotating circular polarization modes which is responsible for a polarization symmetry breaking, whereas the pumping by short pulses reveals a temporal symmetry breaking. We show that both mechanisms can coexist for a range of parameters that we determined numerically and a dynamical interplay is predicted. This work brings further insight into the complex dynamics of nonlinear resonators and will be of high interest for future experimental investigations of such systems, particularly studied in the context of cavity solitons, frequency combs and their applications.

\section{References}

[1] L. Del Bino et al., "Symmetry Breaking of Counter-Propagating Light in a Nonlinear Resonator," Sci. Rep. 7, 43142 (2017).

[2] L. Del Bino et al., "Microresonator isolators and circulators based on the intrinsic nonreciprocity of the Kerr effect," Optica. 5 (3) (2018).

[3] Q. T. Cao et al., "Experimental Demonstration of Spontaneous Chirality in a Nonlinear Microresonator," Phys. Rev. Lett. 118 (3) (2017).

[4] B. Garbin et al., "Buffering optical topological data using passive Kerr resonators," in CLEO, JTu2A.119 (2018).

[5] Y. Xu and S. Coen, "Experimental observation of the spontaneous breaking of the time-reversal symmetry in a synchronously pumped passive Kerr resonator," Opt. Lett. 39 (12) (2014).

[6] M. Haelterman et al., "Polarization multistability and instability in a nonlinear dispersive ring cavity," JOSA B. 11 (3) (1994). 